treatment and management in short training courses

volunteers, trained on brief trauma management courses

O multidisciplinary professionals, to conduct the training.

The training should be modular and based on manuals.

It is important that those who help are also protected by mentoring. If they are to develop into selfhelp communities and local

volunteers are to be trained, then they will be in need of constant help. That should be ensured.
Another important issue is the place of religion, spirituality and religious rituals in enhancing resilience, coping and rebuilding through acceptance and finding some meaning even in suffering and loss. There have been many examples of when type of feeling being empowering.

\section{Governmental liaison - new lives, new cities}

Expert liaison with the government of Pakistan and Kashmir needs to be initiated over social development in view of the emotional state of the communities affected. There needs to be proactive advice on social drift: the risks of drug addiction and misuse, crime, prostitution and so on numbed victims who are untreated can become involved in self-destructive and socially destructive activities).

It is important that new ghettoes are not established in the planned tent cities, that these are only temporary measures and that communities are retained in their structure according to their culture. Graves of parents and ancestors are strong bonds for most people in that area. There is strong attachment to the memory of one's parents in Pakistan and Kashmir. Artificially created communities always develop social problems.

\section{Mentoring and protecting}

It is important that those who help are also protected by mentoring. If they are to develop into self-help communities and local volunteers are to be trained, then they will be in need of constant help. That should be ensured.

To develop as community helpers, the respect and participation of communities are essential. In such times, people need to become protectors of each other, to develop into purposeful individuals and communities.

With such measures in place, services may be able to provide hope to victims and also help local mental health services.

\section{References}

Saraceno, B. (2005) WHO's mental health response to the Asian victims. World Psychiatry, 4, 66-68.

Singh, B. \& D'Souza, R. (2005) Mental health challenges in Sri Lanka from working within the disaster areas. World Psychiatry, 4, 68

Van Ommeren, M., Saxena, S. \& Saraceno, B. (2005) Mental and social health during and after acute emergencies: emerging consensus? WHO Bulletin, 83, 7I-75.

World Health Organization (2003) Mental Health in Emergencies: Psychological and Social Aspects of Health of Populations Exposed to Extreme Stressors. Geneva: WHO

World Health Organization (2005) Briefing Note on Psychosocial/ Mental Health Assistance to the Tsunami-Affected Region. Geneva: $\mathrm{WHO}$

\title{
Senior Volunteers Programme: a visit to Zambia
}

\section{Andrew Sims and Ruth Sims}

Church Farm House, Alveley, Bridgnorth, Shropshire WV15 6ND, UK

\section{Of the 12 million population of Zambia, 50\% are aged 15 or younger. Lusaka, the capital, is home to 3 million, most of whom live in single-room dwellings with minimal amenities.}

Coll he he Board of International Affairs of the Royal College of Psychiatrists initiated the Senior Volunteers Programme in $\mathbf{2 0 0 4}$ following discussion at the Board and planning in a subgroup over the preceding year. The aim was to match requests from other countries for specific psychiatric teaching with the services of senior psychiatrists in Britain. The College has acted as intermediary, inviting requests from countries, Members and Fellows overseas, and setting up a database of psychiatrists offering a range of skills. The concept is simple but all the skill and hard work lies in the detail!

When preparations for the Senior Volunteer Programme were nearly complete, the planning group wished to carry out a pilot scheme using a volunteer. Mr John Mayeya, Mental Health Specialist of the Central Board of Health, Zambia, with Professor Alan Haworth, an Honorary Fellow of the College and Professor of Psychiatry in Lusaka, invited us to contribute to undergraduate teaching in the Medical School of the University of Zambia, participate in the training programme for mental health clinical officers and have seminars and clinical exchange with junior doctors in psychiatry. We were also invited to discuss mental health services and postgraduate psychiatric education.

Of the 12 million population of Zambia, $50 \%$ are aged 15 or younger. Lusaka, the capital, is home to 
3 million, most of whom live in single-room dwellings with minimal amenities. The HIV/AIDS epidemic affects most aspects of everyday life, and virtually every family. The annual death rate from AIDS is still increasing, but we were told that the rate of new infections had peaked and was beginning to decline. Zambia is one of the poorest countries on the poorest continent, and this is everywhere apparent in Lusaka, including mental health services. It was not uncommon to have to buy the petrol before the taxi could take us to the hospital. It is also the most overtly Christian country we have visited, with a penchant for the more energetic Pentecostal denominations, and a church on almost every corner.

Mental health services are mostly delivered through primary care; specialist services are very limited. Patients requiring admission tend to be admitted to local general hospitals. The only specialist mental health facility in the country is Chainama Hills Hospital, on the edge of Lusaka. There are only two trained psychiatrists in the country (as many have left to work elsewhere), and when we were there four other doctors worked in psychiatry in the hospital. Chainama is desperately overcrowded and under-funded, and provision for patients is considerably below an acceptable level. Ward staff, nursing and medical, were kind and caring but struggling under impossible conditions. The numbers of all mental health professional staff are grossly inadequate - doctors, nurses, mental health clinical officers (who could be the linchpin of the whole service) and social workers; other professions are non-existent. Three of the reasons for low staffing, we were told, are:

the haemorrhage of trained nurses and doctors out of the country, especially to Britain

the loss of highly trained and experienced professional staff from the public sector to non-governmental organisations working in Zambia

O advice from the World Health Organization a few years ago that mental health clinical officers should not be recruited directly from school-leavers.

The medical undergraduates were a delight to teach - bright, very interested in psychiatry and keen to use their new knowledge. Unfortunately, as the psychiatric training they receive is largely hospital based, the state of the hospital and the stigma of mental illness discourage most students from working in psychiatry after qualification. The mental health clinical officers were a superb group of dedicated people, with common sense and an enthusiasm for the subject. If only there were an adequate number they could make an enormous contribution to the country; among other roles, they could to a greater extent filter admissions to Chainama, and so lessen the grossly excessive demands currently being made.

There is no formal postgraduate training in psychiatry in Zambia. Medical graduates entering the specialty have to go abroad for further training, and they rarely return. There are 4-year training programmes in medicine, surgery, obstetrics and paediatrics, but not in psychiatry. Equivalent postgraduate training and qualification in psychiatry are essential for the future development of mental health services in Zambia (such programmes already exist in Kenya and Tanzania).

We were in Zambia for most of November 2004. Andrew Sims concentrated on psychiatric teaching on the wards and in the classrooms at Chainama, and in the out-patient department at University Teaching Hospital, Lusaka, as well as giving a few more general lectures. Ruth Sims, as a consultant child psychiatrist, was similarly involved in teaching medical students and in the work at Chainama; she also visited several community facilities for needy children and families.

\section{Was our visit worthwhile?}

From the Zambian point of view, you would have to ask them. For us it was a wonderful experience that we shall always remember. Our grousing, blame culture in Britain could learn a lot from the happiness and fortitude of ordinary Zambians. The problems are huge, but there are also many courageous, charitable people helping in different ways. We felt greatly enriched, and also personally challenged, by our time there.

\section{What could the Royal College of Psychiatrists do for Zambia?}

The College could help enormously in postgraduate education, if this was requested from Zambia. The detail would need to be worked out and any scheme coordinated, but the College's experience of planning and delivering training and the probable availability of short-term teachers not requiring remuneration could help start an invaluable programme.

Obviously, national poverty is a major reason for poor amenities being provided for people with a mental illness. In our opinion, however, stigma is an even greater cause. Zambia needs an organisation for doctors interested in psychiatry to combat stigma at home and to link up with psychiatric societies internationally. It also needs its mental health nongovernmental organisations to be strengthened. The College, through its members, could possibly make a contribution to both.

Finance made available and wisely spent could help to initiate the regeneration of mental health services. The College could use its charitable status to set up an account specifically for improving mental healthcare abroad through donations from members, and Zambia could justifiably benefit from this.
There are only two trained

psychiatrists in the country (as many have left to work elsewhere).

Our grousing, blame culture in Britain could learn a lot from the happiness and fortitude of ordinary Zambians. 